\title{
The Independent Detector Testing Laboratory and the NGST Detector Characterization Project
}

\author{
Donald F. Figer ${ }^{a, b}$, Bernard J. Rauscher ${ }^{a}$, Michael W. Regan ${ }^{a}$, Jesus Balleza ${ }^{a}$, Robert Barkhouser ${ }^{b}$, \\ Louis Bergeron ${ }^{a}$, Gretchen Greene ${ }^{a}$, Sungsoo Kim ${ }^{a}$, Stephan McCandliss ${ }^{b}$, Ernie Morse ${ }^{a}$, \\ Russ Pelton ${ }^{b}$, Tom Reeves ${ }^{b}$, Utkarsh Sharma $^{a}$, P. Stemniski ${ }^{a}$, H. S. Stockman ${ }^{a}$, M. Telewicz ${ }^{a}$ \\ ${ }^{a}$ Space Telescope Science Institute, 3700 San Martin Dr., Baltimore, MD 21218 \\ ${ }^{b}$ Johns Hopkins University, 3701 San Martin Dr., Baltimore, MD 21218
}

\begin{abstract}
The Independent Detector Testing Laboratory (IDTL) has been established by the Space Telescope Science Institute (STScI) and the Johns Hopkins University (JHU), and it will assist the Next Generation Space Telescope (NGST) mission in choosing and operating the best near-infrared detectors. The NGST is the centerpiece of the NASA Office of Space Science theme, the Astronomical Search for Origins, and the highest priority astronomy project for the next decade, according to the National Academy of Science. NGST will need to have the sensitivity to see the first light in the Universe to determine how galaxies formed in the web of dark matter that existed when the Universe was in its infancy ( $\mathrm{z} \sim 10-20)$. To achieve this goal, the NGST Project must pursue an aggressive technology program and advance infrared detectors to performance levels beyond what is now possible. As part of this program, NASA has selected the IDTL to verify comparative performance between prototype NGST detectors developed by Rockwell Scientific (HgCdTe) and Raytheon (InSb). The IDTL is charged with obtaining an independent assessment of the ability of these two competing technologies to achieve the demanding specifications of the NGST program within the $0.6-5 \mu \mathrm{m}$ bandpass and in an ultra-low background $(<0.01 \mathrm{e}-/ \mathrm{s} / \mathrm{pixel})$ environment. We describe the NGST Detector Characterization Project that is being performed in the IDTL. In this project, we will measure first-order detector parameters, i.e. dark current, read noise, QE, intra-pixel sensitivity, linearity, as functions of temperature, well size, and operational mode.
\end{abstract}

Keywords: near-infrared detectors, characterization, NGST, NIR

\section{INTRODUCTION}

The Next Generation Space Telescope (NGST) is the centerpiece of the NASA Office of Space Science (OSS) theme: the Astronomical Search for Origins. The core NGST program to study the origins and evolution of galaxies was recommended by the "HST \& Beyond" committee in 1996. The NGST will need to have the sensitivity to see the first light in the Universe to determine how galaxies formed in the web of dark matter that existed when the Universe was in its infancy $(\mathrm{z} \sim 10-20)$. To achieve this, the NGST is being designed as a cold $(<40 \mathrm{~K})$ telescope located near L2 and optimized for the $1-5 \mu \mathrm{m}$ waveband. Its goal is to detect sources as faint as magnitude 33 (<one photon per second at the detector) to fully exploit its potential.

Sensitive NIR detectors are important for NGST, given the desire to observe the redshifted light from the early Universe. A natural limitation to such observations is presented by sunlight scattered by and thermal emission from interplanetary (zodiacal) dust in the Solar System (see Figure 1). Fortunately, the surface brightness of the foreground emission of that dust is least in the 1-4 $\mu \mathrm{m}$ range, a range in which the apparent spectral energy distributions of high- $\mathrm{z}(\mathrm{z} \sim 1-10)$ galaxies are maximum. Both imaging and spectroscopic observations are required in the NIR, and NIR detectors will be the primary sensors for guiding and establishing/maintaining the wave front performance of the telescope. There are two basic challenges associated with developing these detectors. First, to achieve NGST's planned sensitivity limit, manufacturers must produce detectors that are more sensitive than those flown on previous missions. As illustrated in Figure 1 (left), the total noise resulting from dark current and read noise must be extremely low to assure that NGST imaging is zodiacal light limited. Indeed, the detector will be the dominant noise source for spectroscopy of faint sources, even if we assume optimistic goals for read noise $\left(3 \mathrm{e}^{-}\right)$and dark current $\left(0.02 \mathrm{e}^{-} / \mathrm{s}\right)$. By comparison, the NICMOS HgCdTe detectors on the Hubble Space Telescope have minimum read noise of $18 \mathrm{e}^{-}$(25 samples, up-theramp) and dark current $\sim 0.05 \mathrm{e}^{-} / \mathrm{s}$, while SIRTF InSb detectors are expected to exhibit a minimum read noise of $10 \mathrm{e}^{-}$ (64 samples, Fowler sampling) and dark current $<1 \mathrm{e}^{-} / \mathrm{s}$. The time required to execute the imaging and spectroscopic 
portions of the NGST mission is directly related to basic detector properties like dark current and read noise (Figure 1 right). Second, to provide appropriate spatial sampling for the NIR camera and spectrograph, detector manufacturers must produce many more detectors than have ever been produced before. Figure 2 shows detector effects that have been critical for the HST/NICMOS (Bergeron et al. 1999; Böker et al. 1999; Böker et al. 2000). These effects both reduce data quality and increase the effort required to calibrate and to use the detector.

In part to provide data to the NGST Project, STScI/JHU established the Independent Detector Testing Laboratory (IDTL). The manufacturers are providing NGST prototype detectors to the IDTL for comparative testing. We are developing a set of standardized characterization protocols and making available detailed descriptions of the test setups. Our objectives are to characterize the two competing near-infrared detector types in the parameters indicated in Table 1 as a function of relevant operating conditions using the same procedures, setups, dewars, light sources, targets, electronics, acquisition software, analysis software, and staff. In studying the performance of the detectors using various readout approaches, i.e Fowler sampling, up-the-ramp sampling, differential sampling, off-chip cryogenic amplification, etc., we will identify the approach that yields the best system performance. In addition, we will determine the operational problems that attend the various read modes, including sensitivity to environmental effects, such as a harsh radiation environment and temperature variations induced by the readout approach.
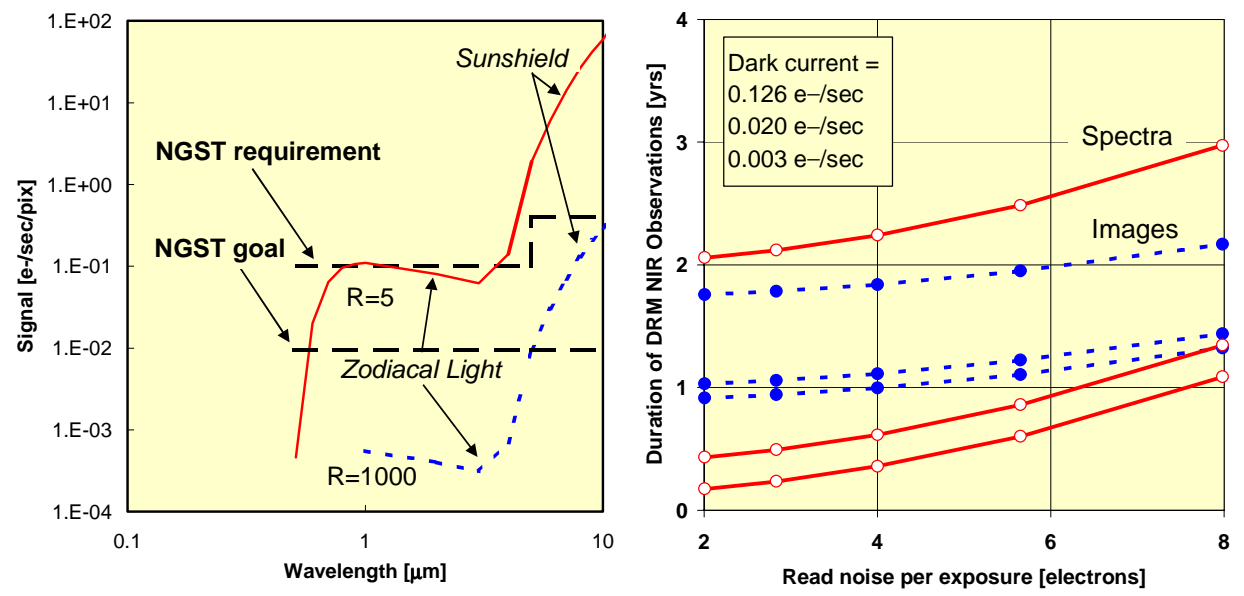

Figure 1.1. NGST needs low noise detectors. (Left) Background rates as a function of wavelength from zodiacal light, and thermal emission and scattered light from the primary and secondary mirrors. (Right) Time required to complete NIR portion of NGST DRM Science versus read noise.

\section{THE IDTL AND THE NGST DETECTOR CHARACTERIZATION PROJECT}

InSb and $\mathrm{HgCdTe}$ detectors are being tested in an ultra low-background environment of the IDTL, using similar test procedures, to measure dark current, read noise, gain, linearity, thermal stability, persistence, absolute/relative QE, intrapixel sensitivity, and short- and long-term radiation effects. Although both detectors are being characterized in similar ways, the controller voltage levels, clock patterns, and operating temperatures are being individually optimized, as guided by our own findings and those of collaborating teams.

A general setup serves the experiments with little modification between experiments for different detector array devices. Blackbody (Model M340 from Mikron Infrared, Inc., Oakland, New Jersey) and monochromator (Model SpectraPro300i from Acton Research, Acton, MA) sources are placed outside the dewar (Infrared Laborabories, Tucson, AZ) upstream from the warm focal plane targets. The pinhole target is controlled by a 3-axis motorized stage (Model ESP300, Newport Corporation, Irvine, CA). There are two cold mechanisms (employing cold motors; Phytron, Waltham, MA) in front of the detector that contain a lens (Janos Technology, Inc., Townshend, VT) for the MTF experiments, filters, and cold blanks for the dark current and latent charge tests. A single detector head is used to provide a light-tight cavity around the detector and it accommodates a cover plate, if necessary, for the dark current tests. 


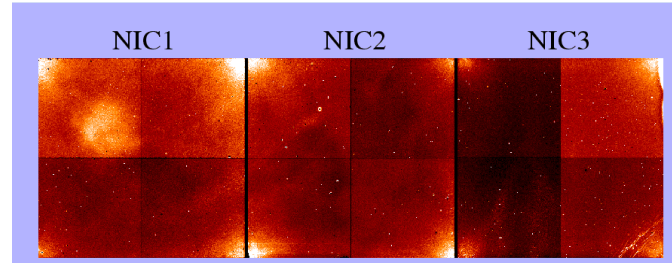

Dark Current

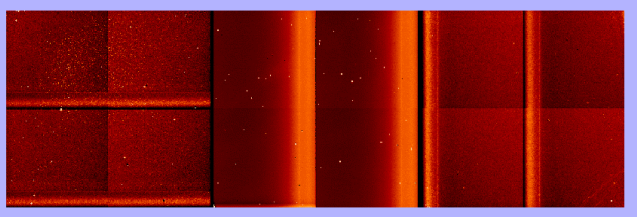

Spatially-dependent bias ("shading")

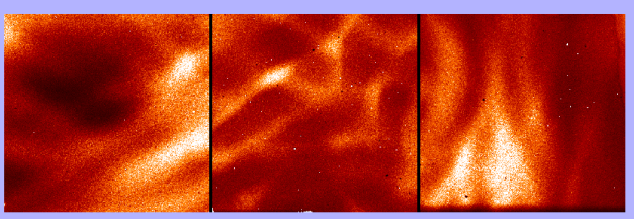

QE Spatial Variations

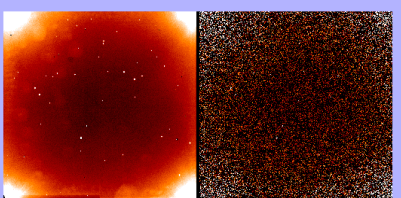

signal

resulting noise

Amplifier (MUX) Glow

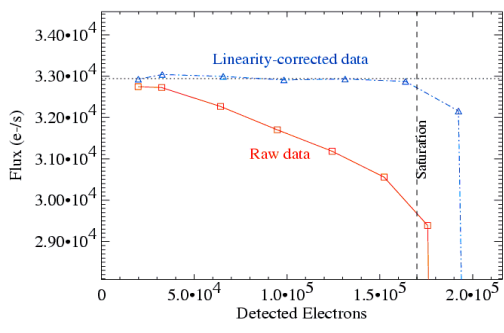

Non-linearity

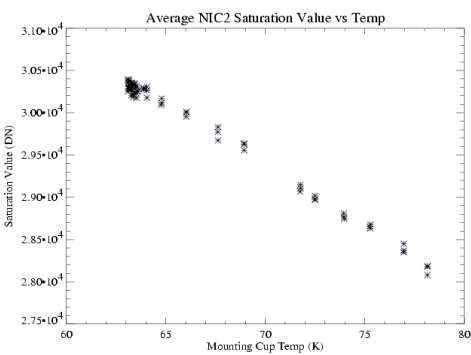

Well-depth vs. Temperature (0.6V bias) $($ gain $=5.4 \mathrm{e}-/ \mathrm{DN})$

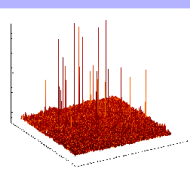

Bad Pixels

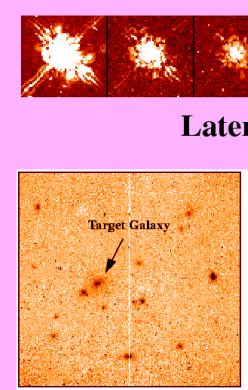

negligible

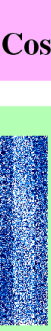

Bias Jumps ("bands")

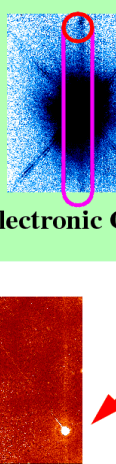

arge Particle Hits

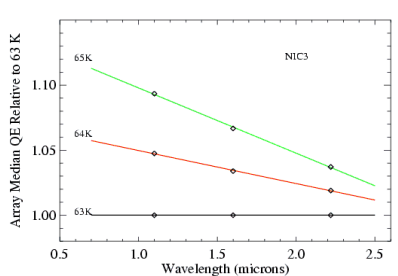

QE vs. Temperature

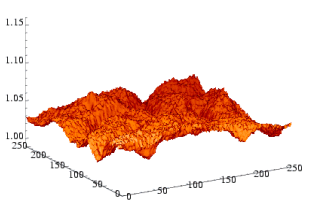

1.1 micron Flatfield Ratio $(64 \mathrm{~K} / 63 \mathrm{~K})$

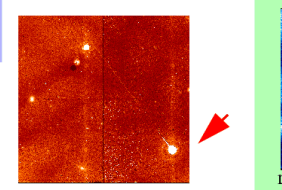

He

A.

Has.

atent Images ("persistence")

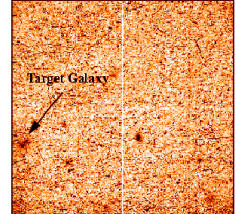

significant Ray Persistence

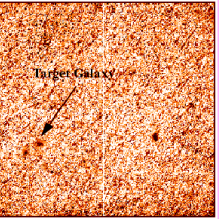

severe

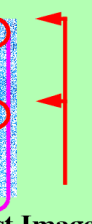

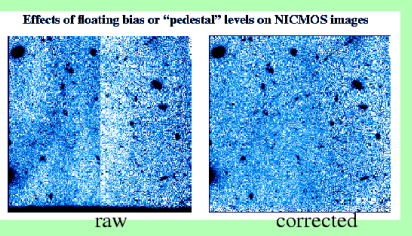

Bias Jumps (“pedestal")

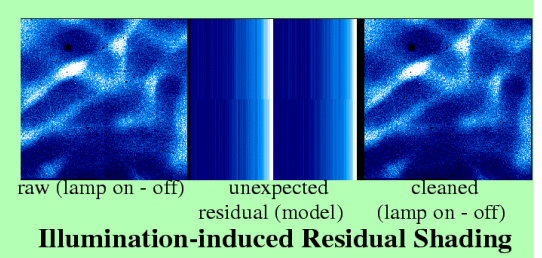

Illumination-induced Residual Shading

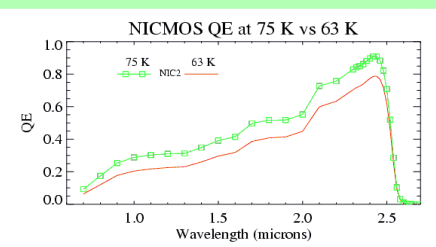

QE vs. Wavelength

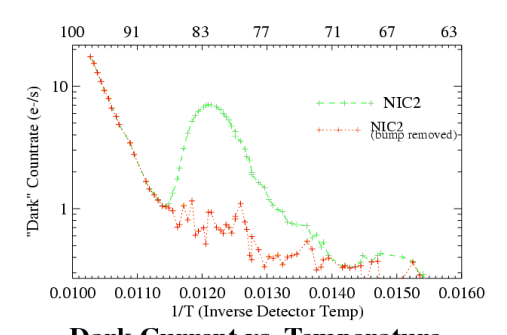

Dark Current vs. Temperature

Figure 1.2. Experience with NICMOS shows that many detector noise sources could limit NGST science. 
Table 1.1: NIR Detector Requirements

\begin{tabular}{|c|c|c|}
\hline Parameter & Requirement & Goal \\
\hline System Noise per image & $9 \mathrm{e}^{-} \mathrm{RMS}$ & $2.5 \mathrm{e}^{-} \mathrm{RMS}$ \\
\hline Read Noise ${ }^{1}$ & $\sim 7 \mathrm{e}^{-} \mathrm{RMS}$ & $\sim 2.1 \mathrm{e}^{-} \mathrm{RMS}$ \\
\hline Dark Current per pixel ${ }^{I}$ & $\sim 0.006 \mathrm{e}^{-} / \mathrm{s}$ & $\sim 0.001 \mathrm{e}^{-} / \mathrm{s}$ \\
\hline$O E$ & $>80 \%$ & $95 \%$ \\
\hline Latent Image & $\sim 0.1 \%$ & 0 \\
\hline Fill Factor & $>95 \%$ & $100 \%$ \\
\hline Radiation Immunity & minimal effect & no effect \\
\hline Frame Time & $<12 \mathrm{~s}$ & $<12 \mathrm{~s}$ \\
\hline$M T F$ & TBD & TBD \\
\hline \multicolumn{3}{|c|}{$\begin{array}{l}{ }^{1} \text { Read noise and dark current have been arbitrarily apportioned, in } \\
\text { quadrature, to meet the system noise requirements and goals in a } 1000 \\
\text { second exposure. There is no specification on the number of reads that can } \\
\text { be used, although the total number of reads must lead to an exposure time } \\
\text { no less than } 1000 \text { seconds. }\end{array}$} \\
\hline
\end{tabular}

\section{HARDWARE AND SOFTWARE DEWAR}

The IDTL hardware system uses a 25" (length) X 16" (diameter) cylindrical dewar made by Infrared Laboratories (Tucson, AZ). We use a CTI Model 1050 cryo-cooler (Helix Technology Corporation, Mansfield, MA) with Model 9600 compressor to provide two cooling stages, one at $\sim 10 \mathrm{~K}$, providing $6 \mathrm{~W}$, and the other at $\sim 60 \mathrm{~K}$, providing $60 \mathrm{~W}$. In addition, we have employed a sinuous bellows tube to provide a LN2 pre-cool capability. We use an outer radiation shield made of aluminum and covered by 10 layers of aluminized mylar (Dunmore Corporation, Newtown, PA) with Dacron mesh interleaved (Apex Mills Corporation, Inwood, NY), in order to reflect and shunt about $20 \mathrm{~W}$ of radiation from the warm dewar walls on to the first stage cooling plate; this superinsulation reduces the temperature of the optomechanical support structure by about $5 \mathrm{~K}$. We use a Lakeshore Model 340 temperature controller (Westerville, $\mathrm{OH}$ ) to sense temperatures at 10 locations and control a heater in the detector thermal path. We are able to control the detector thermal block to $600 \mu \mathrm{K}$ RMS over timescales greater than 10 hours.

\section{OPTOMECHANICS}

The optical design must satisfy the requirements in Table 3.1. The focal plane size is set by size of the detectors that will be tested. At the small end, we must accommodate the Hawaii 1 class of detector, which has $18 \mu \mathrm{m}$ pixels in a $1 \mathrm{~K}^{2}$ package. At the other end of the size range, we must be able to run the InSb $2 \mathrm{~K}^{2}$ detectors, for which we will assumed a maximum pixel size of $27 \mu \mathrm{m}$. So, the active area at the focal plane spans a size from $26.8 \mathrm{~mm}$ to $78.2 \mathrm{~mm}$ from corner to corner of the square field. The object focal plane should be just outside the dewar, how close being dependent on any mechanical structures attached to the targets. The MTF experiment requires a sub-pixel image size. Ideally, we would be able to image an infinitesimally small point of light on to the focal plane. In practice, the object will have finite size, the optical system will induce aberrations, which add to the size and shape of the spot, and the optical system will induce diffraction, which adds size to the spot and imprints the typical Airy pattern on to the spot. The pinhole size will be set by the requirement that it be small enough to produce diffracted light which fully fills the beam and that it be large enough to allow enough light of the test wavelength to pass through the pinhole. Assuming a pinhole size of $5 \mu \mathrm{m}$, then the spot size produced at the image plane will be:

$$
s=\theta F=1.22 \frac{\lambda}{D} F=1.22 \lambda f / \# \quad \mu m .
$$




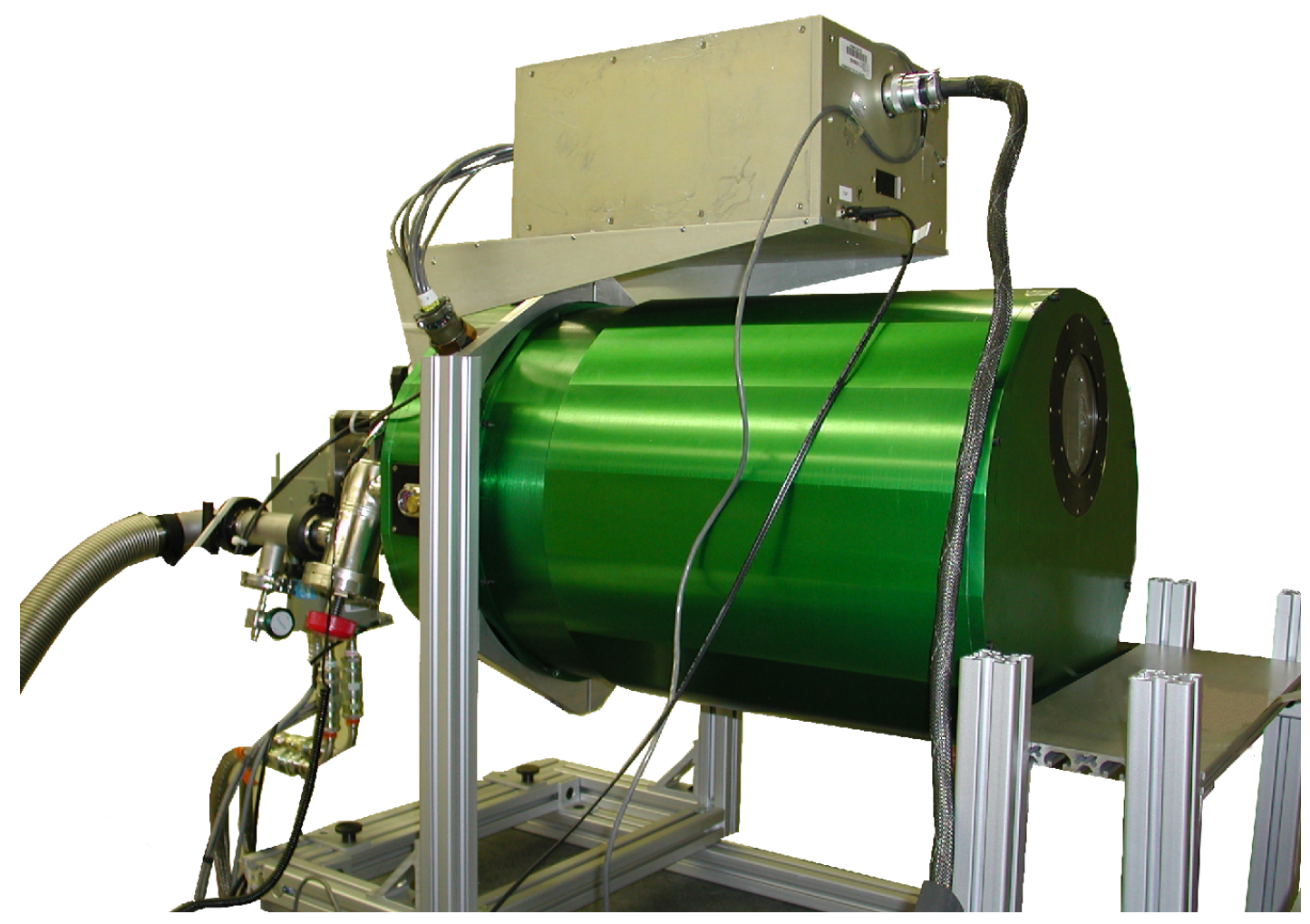

Figure 3.1. IDTL dewar with electronics system on experiment rails.

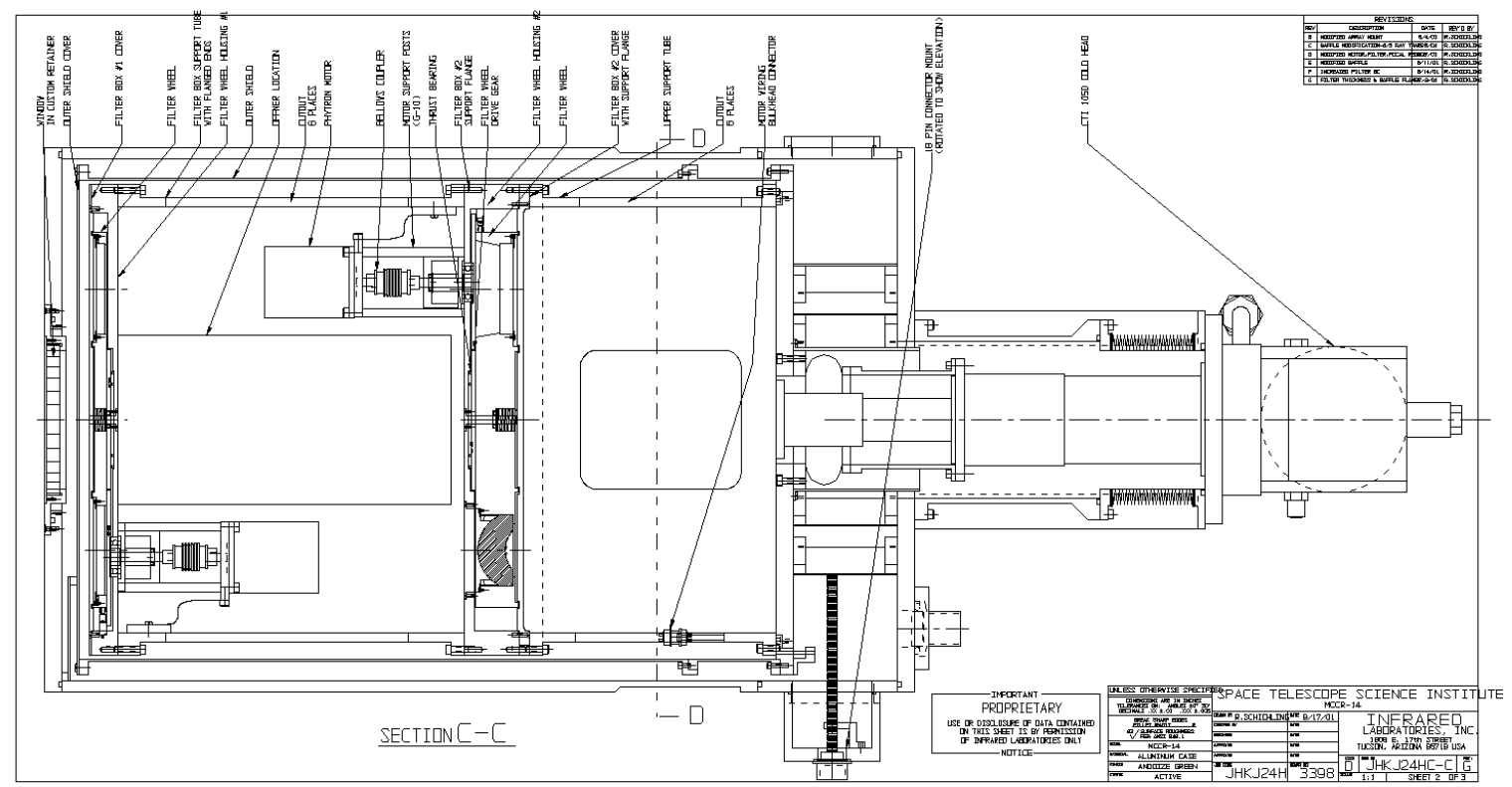

Figure 3.2. Mechanical drawing of cross section of IDTL dewar. 


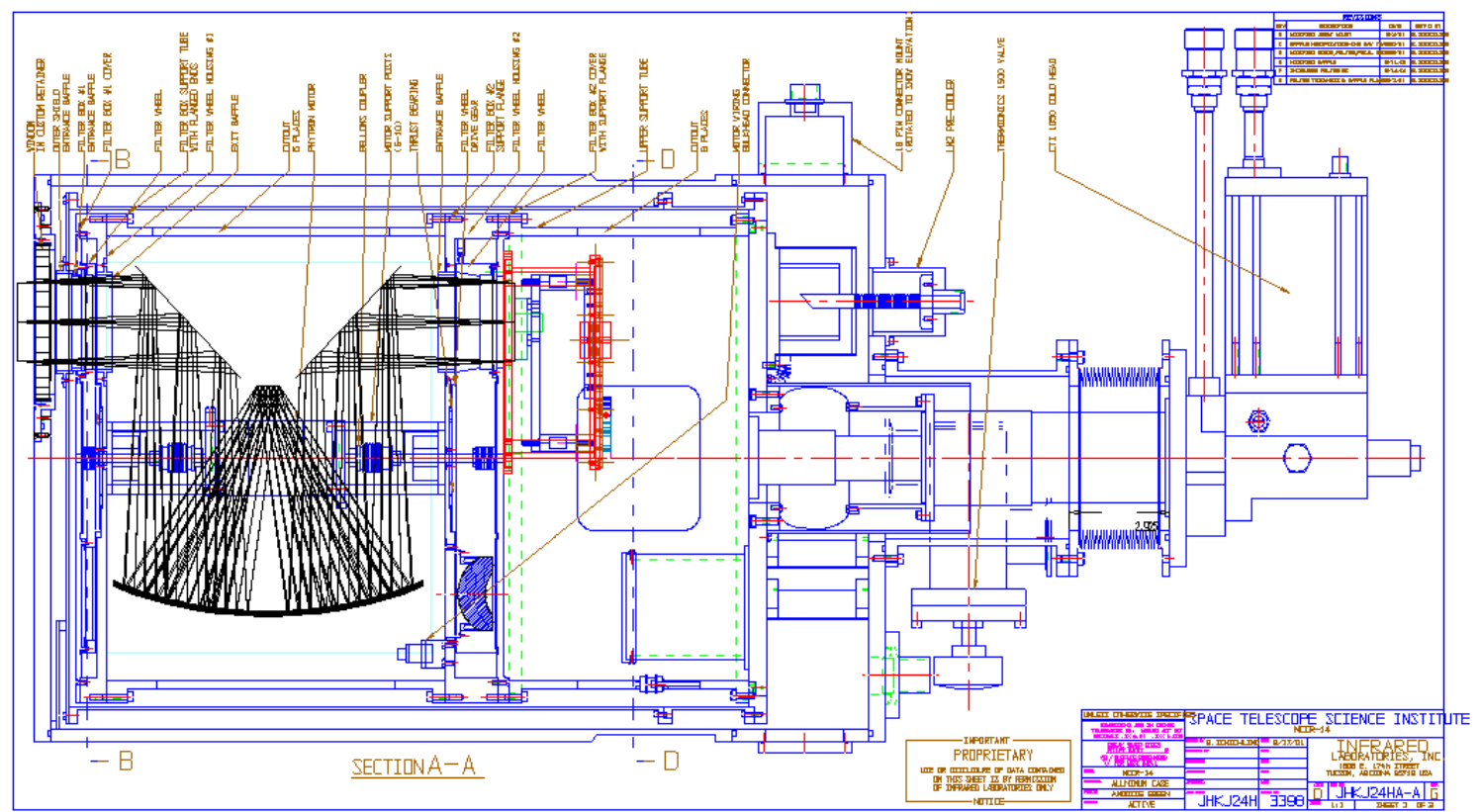

Figure 3.3. Mechanical drawing of cross section of IDTL dewar assembly. The optics with ray trace are also shown.

At $5 \mu \mathrm{m}$, we find a HWHP diffraction-limited spot size of $5 \mu \mathrm{m}$ for $\mathrm{f} / \#=1.5$. Adding the most challenging pixel size, 18 $\mu \mathrm{m}$, we find a spot size that is already $1 / 3$ of the size of the pixel, even without considering the effects of optical aberration or object size. If we add the object size in quadrature to the diffraction spot size, we get an image size of 7 $\mu \mathrm{m}$. This argument becomes much more relaxed for larger pixel sizes and, in particular, shorter wavelengths; although, optical aberrations will begin to dominate at the shortest wavelengths near $0.5 \mu \mathrm{m}$. Given all these considerations, the table lists a requirement on $\mathrm{f} / \#$ and image performance that should be relatively easy to achieve. The wavelength range is set by NGST requirements. The requirements on filters are relatively weak and flow from the desire to obtain measurements in common wavebands. 
Table 3.1: Optomechanical System Requirements

\begin{tabular}{|c|c|}
\hline Parameter & Value \\
\hline Detector temperature & adjustable to $10 \mathrm{mK}$ from $20-80 \mathrm{~K}$ \\
\hline Radiative background at detector & $<0.01 \mathrm{e}^{-} / \mathrm{s} /$ pixel over 0.5 to $5.5 \mu \mathrm{m}$ \\
\hline Maximum system heat load (all sources) & $<50 \%$ capacity of CTI 1050, 3 W @ $20 \mathrm{~K}, 30 \mathrm{~W} @ 60 \mathrm{~K}$ \\
\hline Number of filter positions & 12 (RIJHKLM, focal reducer, hole, blank) \\
\hline Window mounting & modular design \\
\hline Module housings & light tight $\left(0.005 \mathrm{e}^{-} / \mathrm{s} / \mathrm{pixel}\right) \mathrm{w} / \mathrm{mechanical} \mathrm{fiducials}$ \\
\hline Vibration & $<2 \mu \mathrm{m}$ RMS (freq. integrated) at detector \\
\hline Filter absolute positioning (all 3 axes) $)^{2}$ & $1 \mathrm{~mm}$ \\
\hline $\begin{array}{l}\text { Focal reducer absolute positioning } \\
\text { (lateral) }\end{array}$ & $1 \mathrm{~mm}$ \\
\hline $\begin{array}{l}\text { Focal reducer absolute positioning } \\
\text { (piston) }^{3}\end{array}$ & $\mathrm{~d}_{\text {pinhole to window }} /\left(\mathrm{f}_{\text {Offner }} / \mathrm{f}_{\text {focal reducer }}\right)^{2}$ \\
\hline Image performance & $\begin{array}{r}<5 \mu \mathrm{m} \text { FWHM image for } 2 \mu \mathrm{m} \text { object over 10X10 area } \\
\text { near center; otherwise, NR }\end{array}$ \\
\hline Image f/\# & 1.5 \\
\hline Achromaticity & NR, i.e. refocus allowed \\
\hline Wavelength range & 0.5 to $5.5 \mu \mathrm{m}$ \\
\hline $\begin{array}{l}\text { Dewar working volume (length } X \\
\text { diameter) }\end{array}$ & $20^{\prime \prime} \times 14^{\prime \prime}$ \\
\hline Maximum time to change filter position & 10 seconds \\
\hline Mechanical flexure $^{4}$ & $900 \mu \mathrm{m}$ between detector and window \\
\hline $\begin{array}{l}{ }^{1} N R=n o \text { requirement } \\
{ }^{2} \text { This is the requirement on the boresigh } \\
\text { wheel positioning assumes that the intra } \\
\text { filter wheel containing the focal reducer } \\
{ }^{3} \text { For } d_{\text {pinhole to window }}=25 \mathrm{~mm}, \mathrm{f}_{\text {Offner }}=10, \\
{ }^{4} \text { It might be possible to account for this } f\end{array}$ & $\begin{array}{l}\text { any optical element. Using this requirement for filter } \\
\text { sensitivity experiment will be done without moving the } \\
\text { e pinhole has been focussed. } \\
\text { er }=1.5, \text { this equals } 0.5 \mathrm{~mm} . \\
\text { hrough calibration. }\end{array}$ \\
\hline
\end{tabular}

The optical system produces one-to-one imaging on to the detector from the focal plane $10 \mathrm{~mm}$ outside of the window. An Offner (f/10) system is used to form a cold pupil and to re-image the object. The all-aluminum Offner, and its associated optomechanical assembly were designed and machined at JHU/STScI, and Axsys Technologies, Inc. (Rochester Hills, MI) diamond machined the final figure and applied silver coatings. The $\mathrm{CaF}_{2}$ window is $10 \mathrm{~mm}$ thick, with a $110 \mathrm{~mm}$ diameter, and was made by ISP Optics (Irvington, NY). There are two filter locations, one approximately $30 \mathrm{~mm}$ after the object plane, and another the same distance before the image plane. Barr Associates, Inc. (Westford, MA) made the JHKLM and PK50 blocking filters, and CVI Laser Corporation (Albequerque, NM) made the Inconel neutral density filters. A focal reducer lens ( $f / 1.5)$ can be inserted into the beam in order to produce small diffractionlimited spots. The optic is made of $\mathrm{ZnS}$ and has four diamond machined surfaces with unique prescriptions. Portions of the optic are silver coated in order to produce internal reflection. A solid-model rendering is shown in Figure 3.4.

A ray-trace for the central field point, and spot diagrams for central and marginal field points, are given in Figure 3.5. The ray-trace includes the focal reducer, whereas the spot diagrams are made without the focal reducer. The field points are at the extreme points of a $27 \mu \mathrm{m} \mathrm{X} 2048$ field. Note that the geometric spot size is less than a micron for the central field point. The corresponding diffraction-limited spot size is $1.22 * \lambda * \mathrm{f} / \#$, or about $1 \mu \mathrm{m}$ radius (HWHP) for the case with the focal reducer inserted into the beam. 


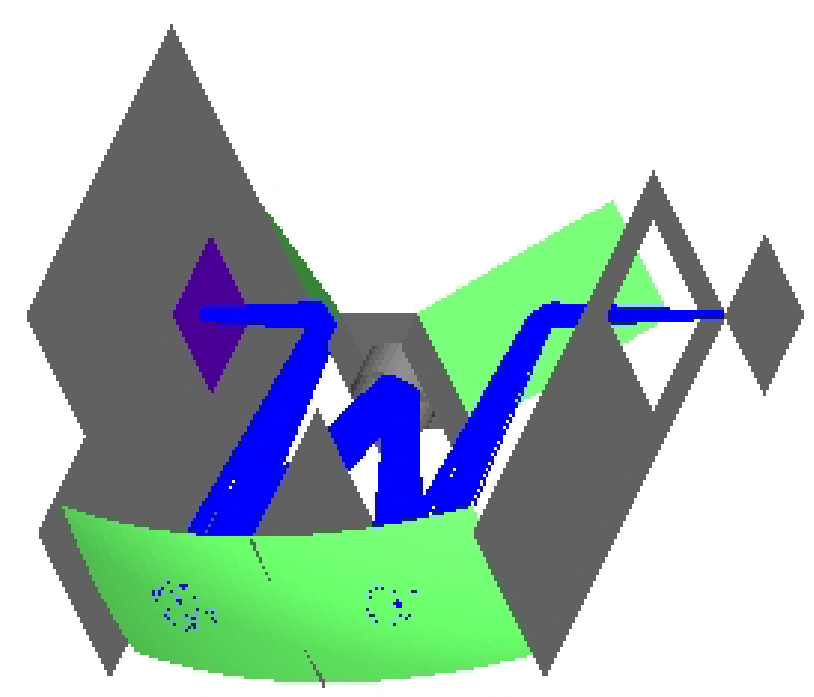

Figure 3.4. Solid model rendering of Offner optomechanical system.
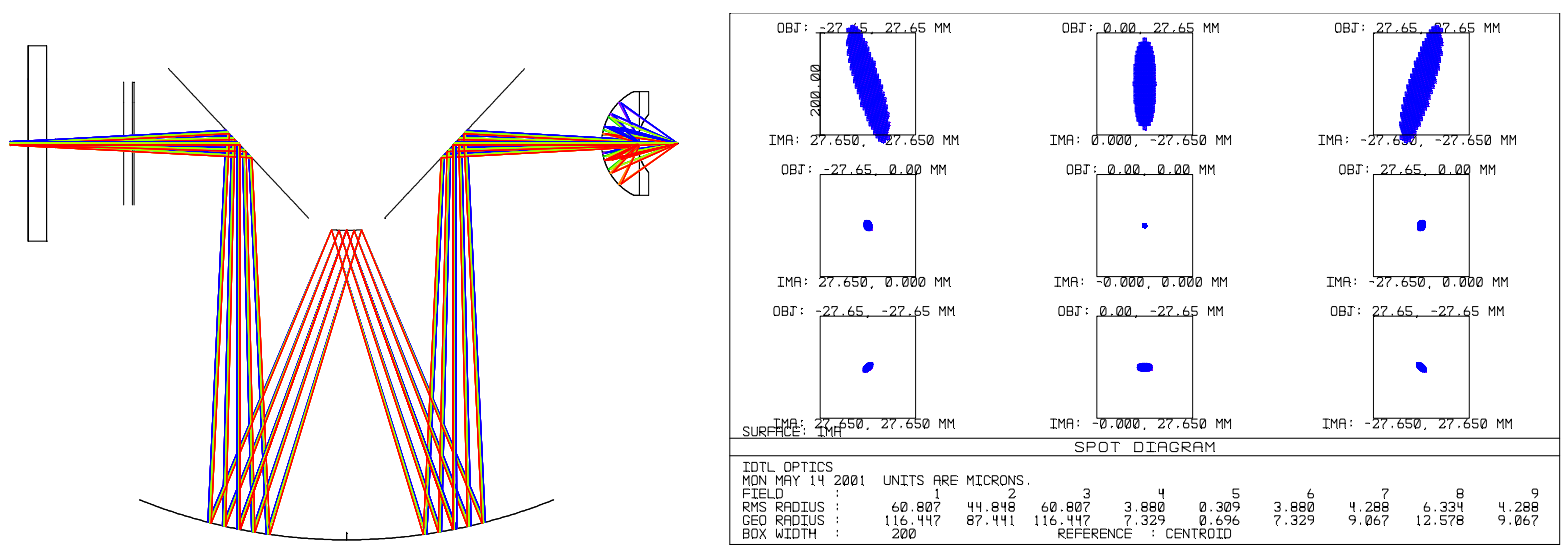

Figure 3.5. Ray trace Offner and focal reducer optical system (left). Spot diagrams for central and extreme field points traced through the Offner-only optical system (right). The extreme field points are located at the sides and corners of a model detector having $2048^{2}$ pixels with $27 \mu \mathrm{m}$ pitch.

We designed and built a light-tight detector head enclosure (Figure 3.6) that interfaces to the dewar, thermal, and electrical subsystems. The detector head enclosure interfaces to the dewar optical subassembly via the lower filter wheel enclosure by an isothermal coupling, ensuring homologous contraction and expansion during cool down and warm up. The enclosure and detector are thermally connected independently to the first and second cooling stage, respectively. Thermal isolation between the detector package and enclosure was an important consideration in selecting materials and interfaces. The detector flex cable passes through the bottom of the enclosure through a two-part connector assembly that captivates a metal dowel which is affixed to the flex cable and back-filled with cryogenic epoxy; we use a custom mounting jig to hold the dowel onto the cable during the epoxy curing process. Mechanical tolerances for the zaxis of the detector head were held to achieve $<0.005$ " cumulative error in positioning the detector surface with respect to the focal reducer lens. The design uses light tight tongue and groove joints with all mechanical part interfaces precisely fabricated using a CNC milling machine. 

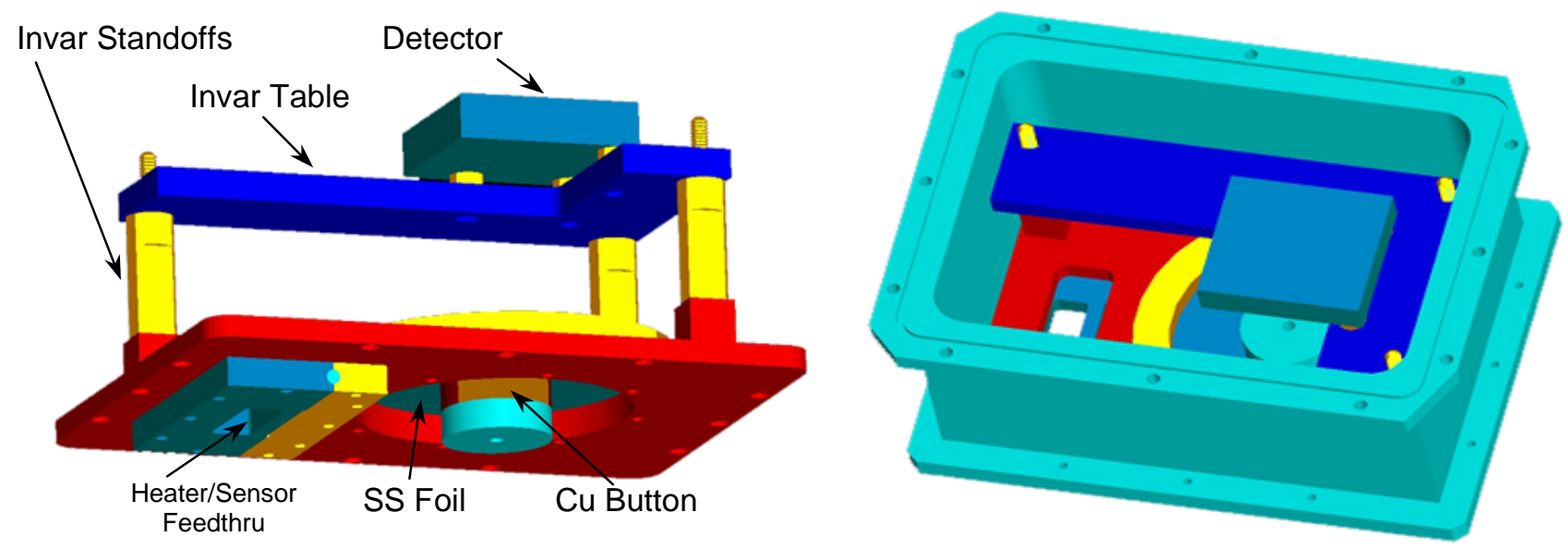

Figure 3.6. Solid model rendering of IDTL detector mounting structure and light-tight enclosure.
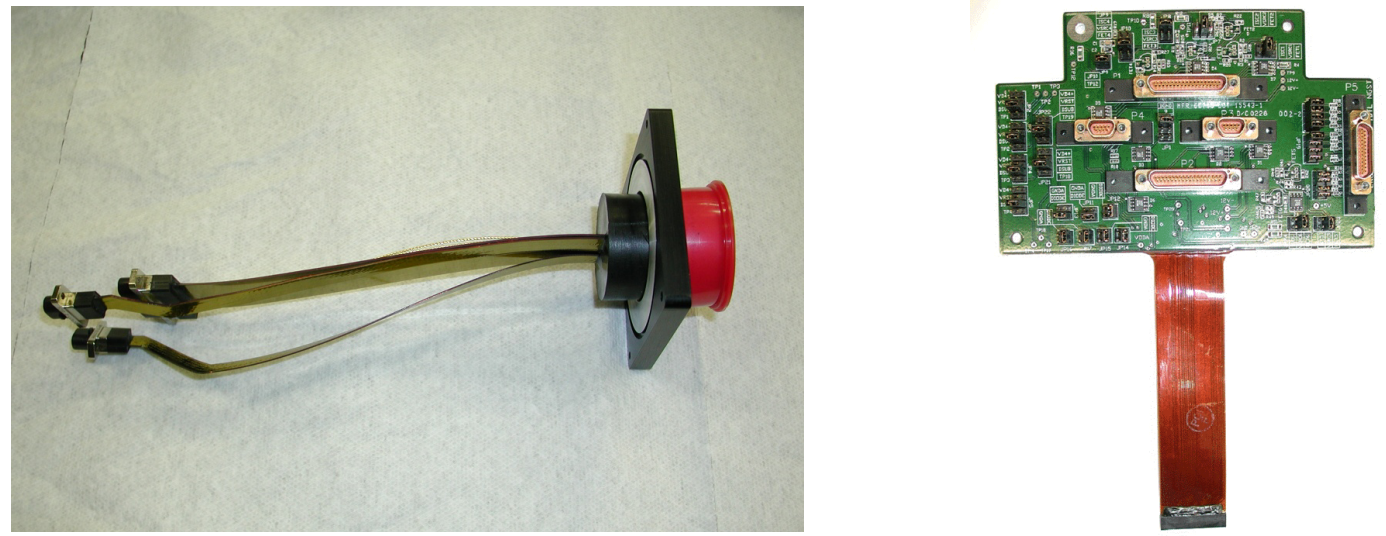

Figure 3.7. "Cold" cable harness (left) and a detector customization circuit (right).

\section{DATA SYSTEM}

The IDTL detector readout system is based on an Astronomical Research Cameras (ARC), Inc. controller (San Diego, CA). The controller is connected to the cable harness (Figure 3.7, left), which is in turn connected to a Detector Customization Circuit (DCC; Figure 3.7, right). The DCC terminates in a single connecter which mates to the vendorspecified connector at the end of the flex cable on the detector package.

The array controller contains two clock driver boards (ARC Document Number CLKDRVIR.R3B), two co-adding video processor boards (ARC Document Number VIDPROCA_R3B.SCH), and a fiber optics timing board (ARC Document Number Gen II TIMING BOARD FIBER OPTIC REV.4B). These cards are built into a completely enclosed box that incorporates cooling fans and a liquid cooling system. ARC designed the sealed box with liquid cooling system to meet the requirements of observatories that need minimal heat dissipation into a dome. Although heat dissipation is not a concern in the lab, maintaining good temperature stability is. We therefore specified the completely sealed system in lieu of a more open box. In practice, we have found that there are enough removable panels in the box to allow us to access all components of the system.

We have four video boards, each with four $1 \mathrm{MHz}$ processing channels containing 16-bit A/Ds made by Datel (ADS937; Mansfield, MA). Eight channels are configured for a gain of 14.3 and eight are configured for a gain of 40. The first 
eight have been used exclusively until this point. They provide a conversion gain of $10.7 \mu \mathrm{V} / \mathrm{ADU}$, referenced to the differential video inputs. The bandwidth is an important parameter affecting the noise. Currently, the $-3 \mathrm{~dB}$ point is set by an RC filter at the video inputs to $80 \mathrm{kHz}$ for the channels used to obtain data presented in this paper. The other twelve channels have a bandwidth of $160 \mathrm{kHz}$.

The cable harness comprises a "warm" cable that is used outside the cryostat and a cryogenic cable used inside the cryostat. Sensitive signals, such as analog biases and video outputs, are carried in a "quiet" cable that is physically separated from the "loud" cable that carries clocks and other non-sensitive signals. The warm cable was fabricated to our specification by Custom Cables Inc. (Laurel, MD). Biases and clocks are carried on shielded 24-gauge 4-conductor cables. Video outputs and a reference output are carried by shielded 24 gauge twisted pair, and a few very sensitive signals (such as the detector substrate) are carried by RG-174 coaxial cable. The warm harness terminates in a 55-pin circular connector (quiet signals) and a 41-pin circular connector (loud signals).

The cryogenic cable consists of laminated ribbon cables that were fabricated to our specification by Tayco Engineering, Inc. (Cypress, CA). Electrically, the cryogenic cable mirrors the warm harness. "Loud" and "quiet" cables are kept physically separate, video outputs and references are carried on 30-gauge copper twisted pair, biases are on single conductor 30 gauge copper wire with a grounded shield wire interleaved every 4 conductors, and a few very sensitive signals are on Lakeshore's copper micro-coaxial cable. The cryogenic cable terminates in four $\mu \mathrm{D}$ connectors that mirror the 4 D-subminiature connectors used in the controller. Taken together, the total length of the warm plus cryogenic cable is about $1 / 2$ meter.

The DCC is a personality card for a particular detector. It differs from the traditional fanout card in that it incorporates a flexible "neck" to simplify interfacing to the rest of the IDTL system. We designed all DCCs, and the Flex Circuit Design Company (San Jose, CA) designed the circuit layout and produced Gerber files that were then used by Pioneer Circuits, Inc. (Santa Ana, CA), for fabrication and testing.

\section{SOFTWARE SYSTEM}

The data acquisition software system is a heavily modified version of the Java-based system (VOODOO) delivered with the ARC controller. We have modified the system to meet the requirements of the IDTL, which differ strongly from that of a detector in an instrument at a ground-based observatory. We need to be able to run automated scripts that take test data over a range of test parameters. To accomplish this automation, we have implemented a scripting interface to the Java system that communicates with the detector. The scripts run in IDL, which gives them full access to IDL's flow and control syntax and allows the script to change and modify test parameters. To date, we have successfully run scripts unattended for more than several days. 
Table 3.2. IDTL Detector Readout Requirements

\begin{tabular}{|c|c|c|c|}
\hline Parameter & Requirement & Goal & Comments \\
\hline Bias Voltage Stability & $\begin{array}{l}\text { Drifts }<130 \mu \mathrm{V} \\
\text { peak-to-peak in } \\
5000 \text { seconds. }\end{array}$ & $\begin{array}{l}\text { Drifts }<38 \mu \mathrm{V} \\
\text { peak-to-peak in } \\
5000 \text { seconds. }\end{array}$ & $\begin{array}{l}\text { Drifts should degrade total noise by }<10 \% \\
\text { when measuring dark current. }{ }^{1}\end{array}$ \\
\hline Per sample nois $e^{2}$ & $\sigma=9.2 \mathrm{e}^{-}$ & $\sigma=2.7 \mathrm{e}^{-}$ & $\begin{array}{l}\text { Electronic rms noise should degrade total } \\
\text { noise by }<10 \% \text { when using } 8 \text { Fowler samples } \\
\text { at the beginning and end of an exposure. }\end{array}$ \\
\hline Crosstalk & $115 \mathrm{~dB}$ & $125 \mathrm{~dB}$ & $\begin{array}{l}\text { Crosstalk due to } \Delta \mathrm{V}=5 \text { Volt transitions on } \\
\text { clocked lines should be negligible compared } \\
\text { to rms noise on bias lines. }\end{array}$ \\
\hline $\begin{array}{l}\text { Cable capacitance (total } \\
\text { on any one lead) }\end{array}$ & $48 \mathrm{pf}$ & $43 \mathrm{pf}$ & $\begin{array}{l}\text { Array outputs should be stable } 10 \mu \text { s after } \\
\text { selecting a new pixel when using a } 20 \mathrm{~K} \text { pull- } \\
\text { up resistor. }\end{array}$ \\
\hline Amplifiers & $\begin{array}{l}\text { System shall all } \\
\text { off-chip output }\end{array}$ & $\begin{array}{l}\text { use of on-chip and } \\
\text { lifiers }\end{array}$ & To separate MUX glow from dark current \\
\hline Temperature stability & $\begin{array}{l}\text { Drifts }<0.26 \mathrm{~K} \\
\text { peak-to-peak in } \\
5000 \text { seconds. }\end{array}$ & $\begin{array}{l}\text { Drifts }<0.076 \mathrm{~K} \\
\text { peak-to-peak in } \\
5000 \text { seconds. }\end{array}$ & $\begin{array}{l}\text { Uncorrected thermal drifts }\left(=500 \mathrm{e}^{-} / \mathrm{K}\right) \text { should } \\
\text { degrade noise by }<10 \% \text { when measuring dark } \\
\text { current. }\end{array}$ \\
\hline \multicolumn{4}{|c|}{$\begin{array}{l}{ }^{1} \text { Using Fourier analysis, we have studied the efficacy of reference pixels located at the ends of row and } \\
\text { columns to reject } 1 / \text { f noise. These models show that uncorrected Fourier components will alias about } 5 \text {-10\% } \\
\text { of the peak-to-peak bias drift on any one bias voltage into RMS noise. } \\
{ }^{2} \text { With input impedance at the chip carrier comparable to an IR array. } \\
{ }^{3} \text { This requirement can likely be met by using } \sim 2 \text { radiation shields with total leaks measuring less than a few } \\
\text { mm in each shield. In addition, we will place a cold metal "blank-off" over the detector as a test of light- } \\
\text { tightness. }\end{array}$} \\
\hline
\end{tabular}

Table 3.3: Control System Requirements

\begin{tabular}{lr}
\multicolumn{1}{c}{ Parameter } & Value \\
\hline Control modes & Interactive, batch (script) \\
\hline User interfaces & GUI (graphical user interface), CLI (command line \\
interface), script
\end{tabular}

In addition to this automation, we have written custom Java interfaces to the various hardware devices that need to be controlled and monitored during the experiments. These interfaces include the filter wheels, thermal controller, various light sources, and a three-axis stage. Every piece of hardware can be controlled either through a graphical user interface, via a command line interface, or from an IDL script.

Because our automated test scripts create large volumes of data for each experiment, we needed to automate the data analysis. But automated data analysis does not work when the various test parameters are only kept in a lab notebook. Therefore, we have adopted the philosophy that all characteristics of the system will be stored in the header of the FITS data files. We map the FITS header keywords to the device-specific parameters using a table driven process that allows us to change the mapping without changing software. Some of the device characteristics that are placed in the header are filter wheel locations, temperatures of all ten of our thermistors, on/off states of all of our light sources, location of the motion controller, bias voltage values on the detector, the current read mode, the name of the file loaded into the timing 
board, and the temperature of our black body source. By having these values in the header directly stored with the data, our IDL scripts can determine what test was being run and, in some cases, automatically generate analysis plots.

\section{PRELIMINARY RESULTS FROM THE NDC}

In this section, we describe preliminary results obtained in the NDC project. Given that we are just beginning the formal detector characterization phase of the project, the data herein should be taken as representative of the type of data that our system can produce, as opposed to being representative of the detectors being tested.

\section{DARK CURRENT}

Dark current is the signal that accumulates in the absence of any light source. In an ideal detector diode, the most important contributors to dark current include charge diffusion, thermal generation-recombination of charges within the semiconductor, and small leakage currents (McLean 1997). With a nominal exposure time of up to 1000 seconds, shot noise $^{1}$ from the dark current is potentially NGST's most important noise source. For example, the near-IR detector requirements are total noise of $9 \mathrm{e}^{-}$. Under these conditions, essentially all of the noise in an $\mathrm{R}>100$ exposure would arise from detector noise, half of which will be from shot noise on the dark current. Our dark current experiment is being performed as a function of temperature.

Figure 4.1 shows the median of data values (ADU) in two images, both obtained using long ramp read sequences, having eight equally spaced reads over a total time of 1000 seconds. The gain for this experiment was approximately 5.5 $\mathrm{e}^{-} /$ADU. Figure 4.2 (left) shows a representative histogram of dark current values. The dark current was measured for each pixel using an equally weighted linear fit to each of the eight data points. The mode of the distribution is 0.017 $\mathrm{e}^{-} / \mathrm{s} /$ pixel with a standard deviation of $0.04 \mathrm{e}^{-} / \mathrm{s} /$ pixel. To date, we have measured dark currents as low as 0.005 $\mathrm{e}^{-} / \mathrm{s} / \mathrm{pixel}$.
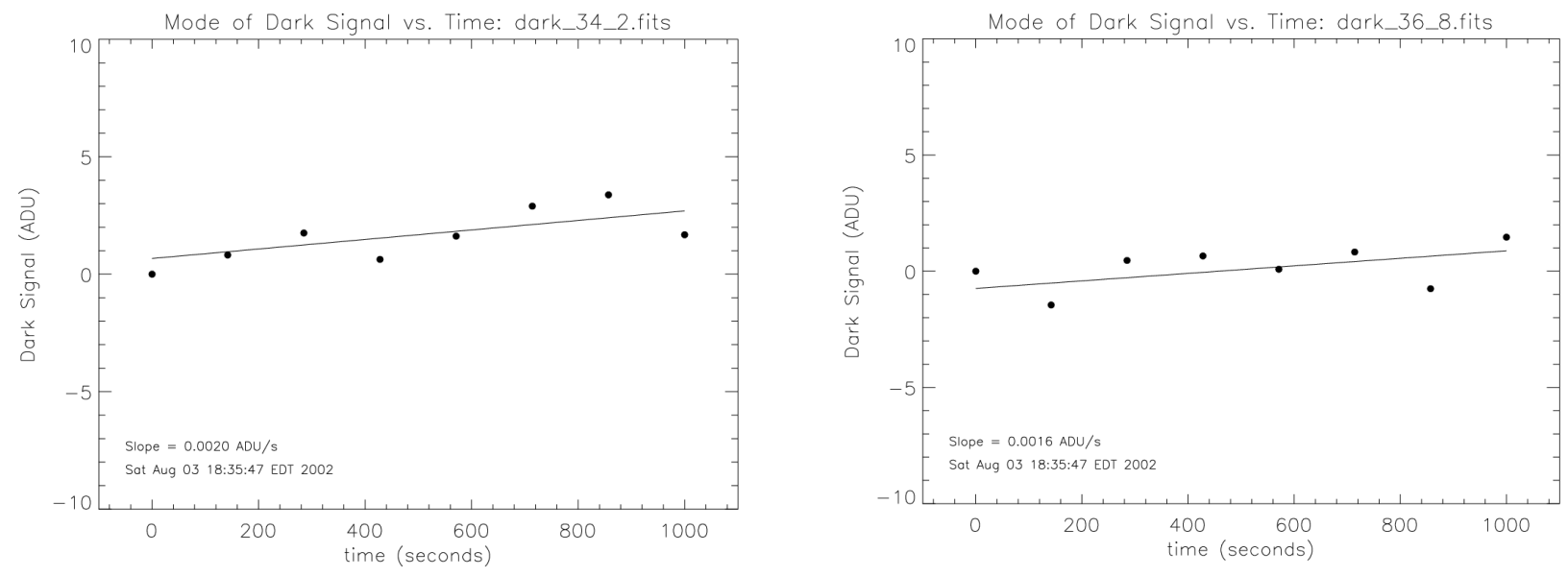

Figure 4.1. Median signal (ADU) versus time for two ramp images in a dark current

Figure 4.2 (right) shows a chi-square distribution for a set of dark current data. A chi-square value is the square of the residual from the fit over the square of the estimated noise, which is assumed to be a summation of the square of the read noise and the square of the number of the electrons generated by the dark. The mean value of the chi-square, 9.9, is close to statistically expected value, 8 , which is the number of readouts. The standard deviation of this distribution, 15 , is also very close to a statistically expected value of 16 . 
The data presented in this section are consistent with a system that achieves the Optomechanical System Requirement of $0.005 \mathrm{e}^{-} / \mathrm{s} / \mathrm{pixel}$; however, it is possible that the system actually exceeds the requirement, if the measurements from the dark current experiments are dominated by true dark current signal.
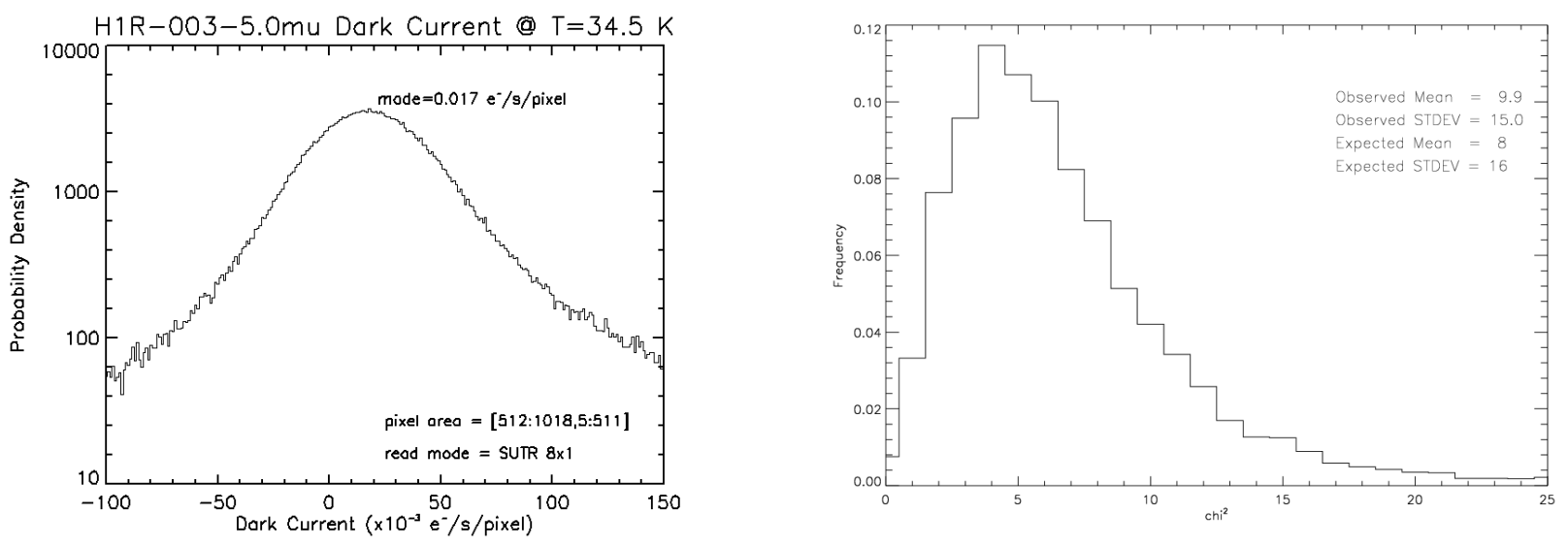

Figure 4.2. Dark current histogram. Distribution of chi-square values from the linear chi-square fit for a dark current experiment.

\section{READ NOISE}

Read noise $\left(\sigma_{\text {read }}\right)$ expresses the uncertainty in measuring the charge in a pixel. Often, it is described as the RMS variation in pixel values over a small patch of the array for a short exposure, or as the RMS variation of all pixel values in a sequence of short exposures. Alternatively, one can measure read noise and system gain ( $\mathrm{g}$; the number of electrons per ADU) using the photon transfer method (Janesick et al. 1987). Using this same experiment, one can also measure linearity.

Read noise is both a property of the detector and of the full readout system. For example, drifts from bias voltages can couple to the output with unity gain. Recognizing the importance of the full readout system in determining the noise, the NGST requirements on total noise are for the sum of contributions from read noise, shot noise from dark current, shot noise from glow, 1/f noise, timing fluctuations, temperature drifts, temperature gradients across the array, and other systematic effects (McCreight et al. 2000). Within the detector hybrid, physical mechanisms contributing to the read noise include resetting the detector capacitance (kTC noise; although this is easily removed using correlated sampling schemes), generation-recombination noise in the semi-conductor and multiplexer, 1/f noise in the multiplexer's FETs, capacitive coupling within the multiplexer and associated sensing circuitry, and inductive coupling from ground through the detector diode and to sense lines.

Read noise can be minimized by reading the same pixel value many times, i.e. via Fowler sampling or through up-theramp sampling. When using Fowler sampling, the relatively long time interval between samples (compared to the time required to digitize a single pixel) averages white noise as well as some 1/f components. In practice, when using multiple non-destructive reads, the read noise diminishes as $n^{1 / 2}$, but shot noise due to glow becomes important for high numbers of samples.

The amount of reduction that can be accomplished using Fowler sampling is limited by the planned 12 seconds it will take to read out the NIR detectors on NGST. Thus, read noise will always contribute significantly to the total detector noise $\left(\sigma_{\text {total }}\right)$. In fact, for some cases it will be the dominant source of noise. For example, cosmic rays may necessitate exposure times as short as 250 seconds (Rauscher, Isaacs, \& Long 2000). In this case, if dark current and read noise 
contribute equally to the nominal system noise in a 1000 seconds exposure, these short exposures would be dominated by read noise. Read noise has an additional impact on the science attainable by NGST through its influence on guiding. Since the frequency of this operation will nominally be $30 \mathrm{~Hz}$, the major source of noise in the determination of the guide star centroid is the read noise (Regan 2000). The short integration times needed for guiding severely limit the number of non-destructive reads that can be used to increase the signal-to-noise ratio. Thus, the inherent read noise of a detector is related to how faint a guide star can be.

Because it is also important to characterize the temporal and spatial relationship of the read noise, we anticipate a series of tests. These include several sets of double-correlated reads with varying times between them, several sets of Fowler-sampled reads with varying spacing, multiple reads of the same pixels and columns (dwell mode) with no or minimal clocking to establish the power spectral distribution of the read noise and the variation in this distribution from pixel to pixel, and differential sampling methods using various reference voltage sources. All of these tests are geared to understand the strength and origin of the readout noise in order to ultimately design an optimum readout strategy for

NGST.

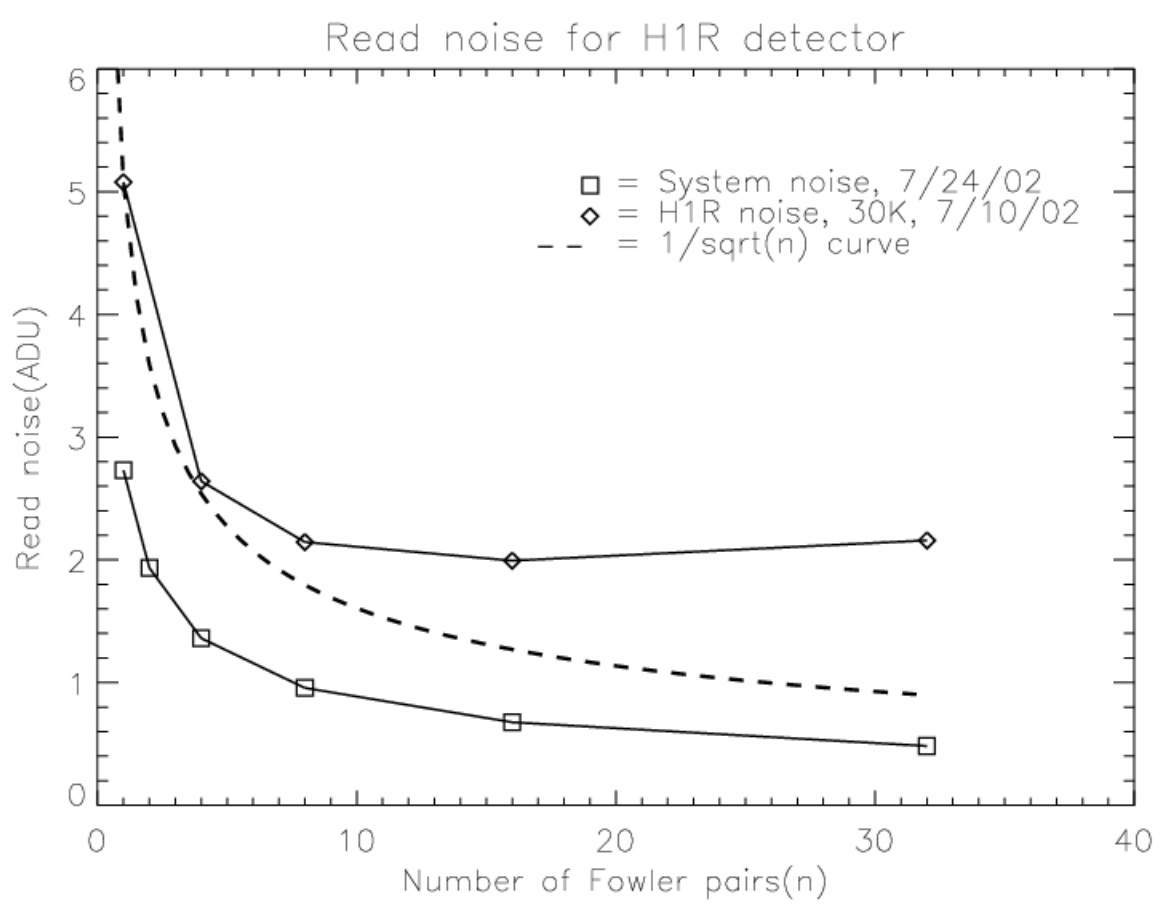

Figure 4.3. Read noise as a function of number of Fowler pairs for the IDTL data system and a Hawaii $1 R$ detector.

Figure 4.3 shows read noise as a function of the number of Fowler pairs (note that correlated-double-sampling is equivalent to one Fowler pair). The IDTL system noise through shorting resistors at the end of all cabling and the DCC is about 2.8 ADU for a CDS frame, and it reduces to 0.5 ADU for $\mathrm{N}=32$. These noise values decrease at the expected rate of $1 / \mathrm{N}^{1 / 2}$. Representative noise measurements for a detector are also overplotted. One can see that the noise deviates from the ideal curve, suggesting the presence of correlated noise sources. Indeed, we will explore the possibility that MUX glow is contributing a significant portion of shot noise for the higher read number data points.

\section{LATENT CHARGE}

Latent charge, or "persistence," is the portion of the signal that is produced by sources in previous images. Anything that liberates charge into the conduction band can result in latent charge, i.e. a bright star or a cosmic ray. Note that latent images are distinguished from spurious sources produced by settling effects in the post-detector analog electronics, i.e. electronic "ghosts" or crosstalk between electronic readout channels.

Latent charge reduces the $\mathrm{S} / \mathrm{N}$ obtainable with an infrared array, and complicates analysis of deep images. It is particularly important in space-based applications, where the sky background is very low, the dynamic range of observed sources is very high, and the radiation environment is intense and variable. Indeed, the sensitivity to this effect was predicted from pre-launch testing of the NICMOS/HST detectors and fully realized on-orbit as elevated dark current after passage through the South Atlantic Anomaly. Even extreme measures, like powering down the NICMOS detectors 


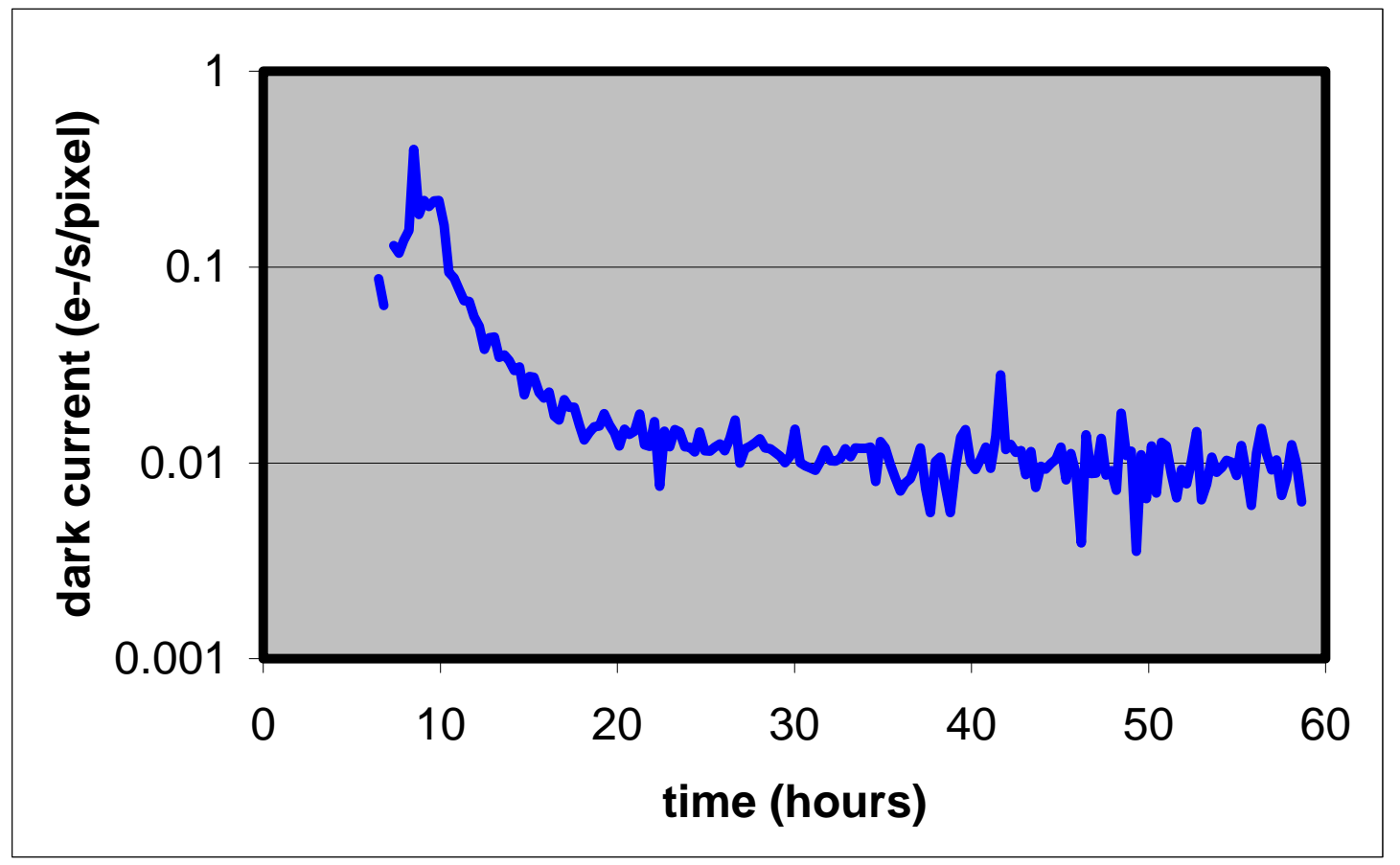

Figure 4.4. Persistent charge decays at a very slow rate. In this experiment, the e-folding time is approximately 12 hours. Note that these data were obtained in the dark current experiment, so we do not know the precise amount of light that hit the detector before the experiment was started. Such information will be collected in the persistence experiment.

during SAA passage, yield no change in the resultant latent charge effect. For NICMOS, latent charge affects data quality and significantly increases the data reduction effort compared to that expected before launch.

It is especially important in the context of NGST to characterize, and attempt to reduce, the effects of persistence. For instance, we have measured the distribution of induced charge from cosmic rays arriving at an altitude of 2600 meters, and estimate an average contribution of $\sim 10^{3} \mathrm{e}^{-}$for a typical cosmic ray hit. Assuming a typical persistence of $1 \%$, that translates to $10 \mathrm{e}^{-}$in trapped charge which would likely leak to the depletion region during the following exposure, assuming that the integration time was longer than 1 minute. This effect is comparable to the system noise requirement for the pixel in question. One could argue that such an effect can be modeled and removed through sophisticated data processing algorithms, but it would be much better to minimize this effect by choosing a detector technology that exhibits low persistence. In any case, persistence needs to be measured and understood before detector down-select for the NGST to be successful.

Our experiment will be conducted with an external monochromator light source. We will calibrate the light source by measuring the induced charge rate on the detector as a function of wavelength, using calibrated filters. A set of baseline data will be obtained under no illumination using a sequence of non-destructive reads to measure dark current, residual bias, and cosmic ray induced charge. The array will then be flooded with varying light levels from the internal light source up to 100X the detector full well. The pupil wheel will then be commanded to insert the cold blank, and dark frames will be obtained using the same exposure parameters as those used to obtain the baseline data. The two sets of data will then be subtracted from each other so that only latent charge remains. The differences give latent charge release as a function of time and incident light level. This test will be repeated for a range of detector temperatures. In addition, we will try different operating modes in order to test mitigation techniques and to distinguish the fraction of the induced charge that can be eliminated by annealing and multiple resets. We will repeat all experiments using an external laboratory radiation source (Co-60 or Americium) in order to determine latent charge effects due to cosmic ray hits. 


\section{ABSOLUTE QUANTUM EFFICIENCY}

Absolute quantum efficiency is the photoelectron production rate as a function of incoming photon flux. In general, the response of any solid-state detector is proportional to the incoming photon flux; however, different pixels have different gains ( $\left.\mathrm{e}^{-} / \mathrm{photon}\right)$. At short wavelengths, input photons can produce more than one photoelectron because the energy of the photon is far in excess of the detector material band gap.
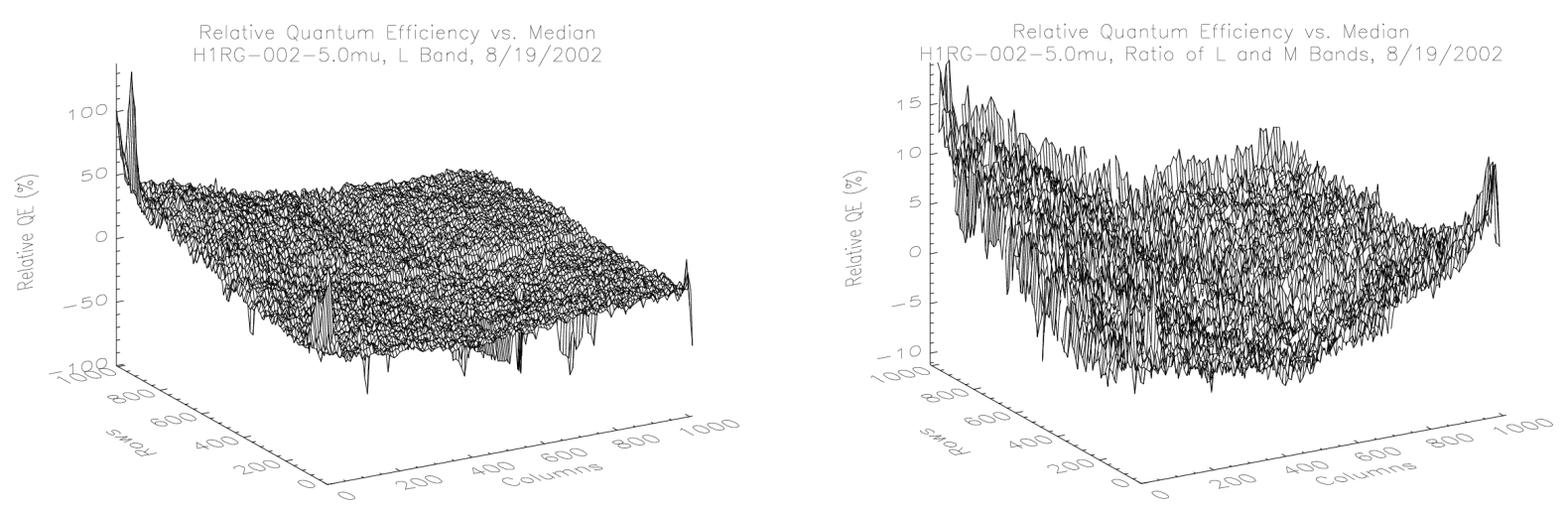

Figure 4.5. Relative $Q E$ (left) in the $L$-band $(3.6 \mu \mathrm{m})$. Ratio of relative $Q E$ maps for $L$ and $M$-bands $(4.8 \mu \mathrm{m})$.

Pixel-to-pixel variations in quantum efficiency (relative quantum efficiency) can result from differences in the crystal structure of the light sensitive material that are created when the crystal is grown. Other causes are geometrical and can be traced back to the manufacturing process, such as variations in the effective detector area and collecting volume (depletion region), variations of integration capacity, and changes in the thickness of the substrate and/or in the antireflection coating. In principle, the effects of relative quantum efficiency can be calibrated and corrected, as long as the detector performance remains stable over time. Several phenomena like cosmic rays, thermal cycling, and temperature drifts (also related to variations of the radiative thermal background) may change the relative quantum efficiency of a detector. Thus, excellent quantum efficiency stability under these varying conditions is required.

We are measuring relative quantum efficiency over the specified NGST bandpass ( 0.6 to $5 \mu \mathrm{m})$ and detector temperature of 30 and 40 Kelvin. An incandescent lamp is used as a light source, and the source flux is passed through the monochromator and integrating sphere (Labsphere, North Sutton, NH). Broadband filters located inside the dewar are used for order blocking. Figure 4.5 (left) shows a relative $\mathrm{QE}$ map over a $1 \mathrm{~K}^{2}$ detector in the $\mathrm{L}$ band. Note that one corner has QE that deviates by up to $40 \%$ with respect to the median for the array. The right panel in that figure shows that the QE map varies considerably as a function of wavelength between the $\mathrm{L}$ and $\mathrm{M}$ bands.

Absolute quantum efficiency generally varies by $\pm 20 \%$ over an array and can be calibrated in science data. However, as with many detector properties, it can vary as a function of temperature, so thermal stability is important. This variation jeopardizes achieving the nominal DRM requirement of $1 \%$ photometric accuracy, so QE variations of less than this amount are required between calibrations, i.e. week timescales, at least.

A flux-calibrated and uniform field is needed to measure absolute quantum efficiency, for which we use the blackbody source, which can be controlled to provide accurate and stable temperatures to within $0.1 \mathrm{~K}$. The detector is illuminated through the Offner relay and calibrated broadband filters. By this method we are able to reliably measure both the absolute quantum efficiency and the change in quantum efficiency with wavelength. 


\section{MTF AND INTRA-PIXEL SENSITIVITY}

The Modulation Transfer Function (MTF) is the Fourier transform of the spatial response of the detector. The primary reasons for deviations from an ideal MTF are crosstalk between the pixels and intra-pixel sensitivity variations. At nearinfrared wavelengths, photon absorption occurs at depths in the detector material that are significant relative to the width of a pixel. Because of this, a photoelecton experiences a three-dimensional random walk until it recombines to the valence band or reaches the edge of a depletion region. Thus, a photoelecton generated under one well may eventually land in an adjoining well (crosstalk), blurring the image and reducing the MTF. In addition, the photoelectons generated near pixel boundaries have to travel larger distances to reach the depletion region and thus have a higher probability of being lost through recombination. This variation in detection probability leads to a variation in intra-pixel sensitivity.

The effects of crosstalk and intra-pixel sensitivity variations become important when the detector is operated in an under-sampled regime. In this case, a high inter-pixel crosstalk will degrade the image that could be reconstructed via dithering, while a large variation in intra-pixel sensitivity will lead to photometric errors. The right hand panel of Figure 4.6 shows photometric errors encountered on NICMOS when camera 3 was used with the F160W filter. When the nearinfrared camera of the NGST is operating at its blue limit, it will under-sample the PSF by a factor greater than three and thus concentrate a large fraction of the light of a point source onto a single pixel. In this regime, low values for the crosstalk and intra-pixel sensitivity variation will be desirable. Unfortunately, a very high MTF requires very small diffusion lengths and well separated depletion regions. These requirements work against the desire to have small pixels, high QE, and the absence of dead space between pixels, characteristics that are desirable for performance and manufacturing considerations. Evidently, these contrary desires require that accurate comparative testing be done, and a significant trade-off be made in the detector design and selection phase.

Our efforts to measure intra-pixel sensitivity are summarized in Sharma et al. (2002).
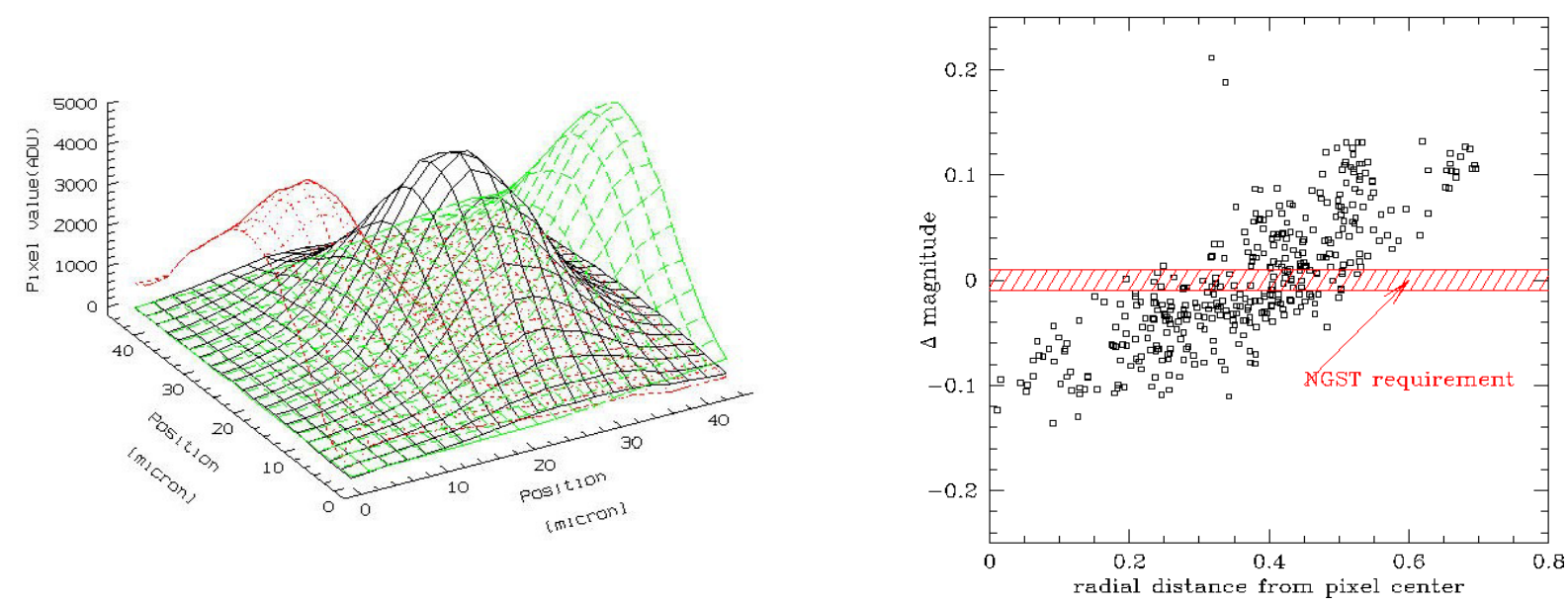

Figure 4.6. DRM-quality photometry (1\%) will be difficult to obtain according to measurements of intra-pixel sensitivity. (Left) Intra-pixel sensitivity of $\mathrm{HgCdTe}$ to a point source measured by Finger et al. (2000). (Right) NICMOS magnitude errors as a function of intra-pixel source location (Stiavelli, private communication). 


\section{ENVIRONMENTAL EFFECTS RADIATION}

High energy charged particles pose a threat to spaceborne electronics and detectors. The flux of these particles can be reduced, but not eliminated, by shielding. An obvious manifestation of cosmic ray hits in a detector is a spurious signal that results when electrons are excited into the conduction band. Based on our analysis of IRAC data (Rauscher, Isaacs, $\&$ Long 2000), we find that a typical hit raises $\sim 5$ pixels 5 sigma above the noise floor. Average hits on the NICMOS $\mathrm{HgCdTe}(40 \mu \mathrm{m}$ pixels) raise $\sim 2$ pixels significantly above the noise floor, however median hit intensity in accelerator data is much higher than on-orbit.

We have planned radiation testing using a $1 \mu \mathrm{C} \mathrm{Cf-252} \mathrm{(Isotope} \mathrm{Products)} \mathrm{button} \mathrm{source.} \mathrm{We} \mathrm{will} \mathrm{obtain} \mathrm{long} \mathrm{dark}$ frames using the up-the-ramp read mode, during and after, exposure to the alpha particles from the radiation source. We will flag hits and monitor subsequent persistence for primary and neighboring pixels.

\section{TEMPERATURE EFFECTS}

The requirement on detector operating temperature is somewhat flexible and comes from the desire to operate the detectors with as little cooling power as possible. The detector technologies are advancing at a rapid pace, and it is too early to know optimal operational temperatures. The present NGST architecture demands that the observatory thermal design be able to provide a $37 \mathrm{~K}$ stage for the NIR detector.

Separate from the issue of determining the optimal temperature, one must know the thermal stability that is needed to achieve the performance requirements in Table 1, given a realistic NGST environment, i.e. changes in telescope pointing and spacecraft thermal exposure. Such effects can be important, given that the most challenging programs require photometric stability on the order of $0.1 \%$. Unfortunately, very small temperature variations can induce large DC offsets in the measured signal and variable "shading" amplitudes in the HgCdTe detectors (Böker et al. 2000). This induces an imprint of the QE pattern onto the reduced image, inducing several hundred electron variations across the NICMOS detector for $100 \mathrm{mK}$ drift within a single HST orbit. Variable shading is a much greater problem. We expect to see residual bias in the target minus bias frame on the order of $0.3 \mathrm{e}^{-} / \mathrm{mK}$ due to thermal drifts. Lallo et al. (1998) find that the NGST sunshield-to-instrument module thermal circuit produces about $20 \mathrm{~mW}$ of parasitic heating in the instrument module, and that this load is a function of telescope pointing. Of course, this is separate from the effects of detector selfheating. A change of a few $\mathrm{mW}$ of heating power can induce temperature changes on the order of $30 \mathrm{mK}$, or $30 \mathrm{e}^{-}$in variable DC offset and $10 \mathrm{e}^{-}$in variable "shading," or much greater than the whole NGST system noise requirement. An effect of similar magnitude can be seen in the InSb detectors, where Finger et al. (2000) measured a drift of $1.7 \mathrm{e}^{-} / \mathrm{mK}$ for dead pixels. Rauscher et al. (2002) summarize preliminary work we have done in using reference pixels to ameliorate the effects of mid- to low-frequency noise. Similar techniques should be directly transferable to reducing effects induced by temperature affects, assuming they are at similar frequencies.

\section{OPTIMAL USE STUDY - HOW WOULD EITHER DETECTOR BE OPERATED FOR NGST?}

Ultimately, for the NGST, the operating modes will be designed to optimize scientific performance under system and financial constraints. In particular, the number, speed, and duration of readouts will affect the observing efficiency as well as the overall thermal balance of the ISIM (direct on-chip dissipation, analog electronics power dissipation, and the parasitic heating by the wire bundles). Thus, the choice and design of operating modes will be an exacting engineering exercise that must leave some flexibility for on-L2 experience. One of the key goals of the characterization effort is to provide the low-level performance characteristics needed to model and design optimum operating modes, test a specific subset of these modes to validate the models, and compare the two NIR technologies with their optimum readout modes. We do not expect the detailed optimum operations for InSb and $\mathrm{HgCdTe}$ to be identical. Characteristics that will affect the design of the readout scheme will include: relative values of read noise and dark current, induced signal from clocking and readout, white-noise levels, true pixel-level 1/f noise (usually unmeasureable), sensitivity to thermal drifts (read-induced and external) and externally-supplied bias levels, availability of reference signals (per pixel, per column, per frame), and the uniformity of these sensitivities across the device. 
Not withstanding engineering and financial constraints, the optimum operating modes for the NGST will depend on the typical background and maximum integration time (cosmic ray effects). In general, they may consist of a combination of up-the-ramp (for cosmic ray rejection), multiple (Fowler) samples (for thermal drifts), with long "integrations" per pixel (for white noise reduction). The spacing per ramp-sample, number of Fowler samples, and integration period will be designed to maximize the overall SNR or minimize the RMS variance including cosmic ray effects and the loss of integration time due to readouts. Part of this study will be the creation of a simple model for each device that includes the principle read-noise effects and their amelioration. Given a background level and cosmic ray flux, the model will

produce a range of operating modes with comparable signal-to-noise performance.

\section{ACKNOWLEDGEMENTS}

The material in this paper is based upon work supported, in part, by NASA, under award No. NAG5-10430.

\section{REFERENCES}

Ando, K., Estrada, A. D., Love, P. J., Smith, M. S., \& Sparkman, K. P. 2000, "Infrared detectors for ground-based and space-based astronomy," SPIE, 4008-159

Barth, J. L., \& Isaacs, J. C. 1999, "The Radiation Environment for the Next Generation Space Telescope," NGST Project, internal document

Bergeron, L. E., \& Najita, J. R. 1998, "Cosmic Ray and Photon Image Persistence in NICMOS, and Ways to Reduce its Effects", BAAS, 193, \#36.06

Bergeron, L., Böker, T., Bacinski, J., \& Mazzuca, L. 1999, "Observed Characteristics of the HST/NICMOS Dark Current During the end-of-life Warm-up," STScI, Baltimore, MD, internal document

Böker, T., Bacinski, J., Bergeron, L. E., Holfeltz, S. T., Gilmore, D., Monroe, B., \& Sosey, M. 1999, "Analysis, results, and assessment, of the NICMOS warm-up monitoring program," NICMOS ISR-99-001, STScI, Baltimore, MD

Böker, T., Ferro, T., Holfeltz, S. T., Hubbard, P., Jarrell, D., Monroe, B., O'Neil, E., Schneider, G., \& Sosey, M. 2000, "NICMOS Dark Current Anomaly: Test Results," ISR-00-002, STScI, Baltimore, MD

Daou, Doris, \& Skinner, Chris, "Persistence in NICMOS: Results from On-Orbit Data," STScI Instrument Science Report NICMOS-97-024, 12 September 1997

Figer, D. F. 1998, "NIRSPEC Optics Design Note 26.00: NIRSPEC Optomechanical System Acceptance Test Plan", NIRSPEC project internal document

Finger, G., Biereichel, P., Mehrgan, H., Meyer, M., Moorwood, A. F. M., Nicolini, G., \& Stegmeier 1998, "Infrared Detector Development Programs for the VLT Instruments at the European Southern Observatory," SPIE, 3354, 87

Finger, G., Biereichel, P., Mehrgan, H., Meyer, M., Moorwood, A. F. M., Nicolini, G., \& Stegmeier, J. 1999, "Experience with near IR detector arrays used for low background ground based applications," NGST Detector Workshop April 20-21, 1999, STScI, Baltimore, MD

Finger, G. , Biereichel, P., Mehrgan, H., Meyer, M., Moorwood, A. F. M., Nicolini, G., \& Stegmeier, J. 2000, "Performance of large format $\mathrm{HgCdTe}$ and InSb arrays for low background applications," SPIE, 4008-143

Fowler, A. M., \& Gatley, I. 1990, ApJ, 353, 33

Fowler, A. M., Gatley, I., McIntyre, P., Vrba, F. J., \& Hoffman, A. W. 1996, “ALADDIN: the 1024x1024 InSb arraydesign, description, and results", SPIE, 2816-150

Garnett, J. D., \& Forrest, W. J. 1993, "Multiply sampled read limited and background limited noise performance," SPIE, 1946,395

Geithner, P. 2000, "Detector Temperature Requirement for NGST Near Infrared (NIR)", NGST Project Memo

Hall, D. N., \& Hodapp, K. 2000, "Characterization of $\lambda_{\mathrm{c}}=5 \mu \mathrm{m} \mathrm{Hg:Cd:Te} \mathrm{arrays} \mathrm{for} \mathrm{low-background} \mathrm{astronomy,"} \mathrm{SPIE,}$ 4008-142

Hodapp, K.-W. et al. 1996, "The HAWAII Infrared Detector Arrays: testing and astronomical characterization of prototype and science-grade devices", New Astronomy, vol. 1, no. 2, 177

Janesick, J. R., Elliott, T., Collins, S., Blouke, M. M., \& Freeman, J. 1987, Optical Engineering, 26, 692 
Lallo, M., Bely, P., \& Petro, L. 1998, "Thermal Considerations for a Passively Cooled NGST", in The Next Generation Space Telescope: Science Drivers and Technological Challenges, Liege, Belgium

McKelvey, M., McMurray, Jr., Robert E., Johnson, Roy R., McCreight, Craig, \& Castaneda, Carlos, "SCA30218 Proton Tests at the UC Davis Cyclotron," October 1999

McKelvey, M., McMurray, Jr., Robert E., Johnson, Roy R., McCreight, Craig, \& Castaneda, Carlos, "Recent Proton Tests at the UC Davis Cyclotron," May 1998

McCreight et al. 1999, "Recommendations of the NGST Detector Requirements Panel"

McLean, I. S. 1997, "Electronic Imaging in Astronomy: Detector and Instrumentation," John Wiley \& Sons: Chichester, p. 222

Najita, J., Dickinson, M., \& Holfeltz, S., “Cosmic Ray Persistence in NICMOS Data," STScI Instrument Science Report NICMOS-98-001.

Rauscher, B. J., Isaacs, J., \& Long, K. 2000, "Cosmic Ray Management on NGST: The Effect of Cosmic Rays on Near Infrared Imaging Exposure Times", STScI, Baltimore, MD

Regan, M. W. 2000, "Guiding with NGST NIR detectors," STScI, Baltimore, MD

Pipher, J. et al. 1999, "Radiation Testing of InSb Array on CRC 744 Multiplexer," Presentation to the IRAC Team 9/15; Revised 11/99

Seltzer, S. M. 1994 "Updated Calculations for Routine Space-Shielding Radiation Dose Estimates: SHIELDOSE-2," NIST Publication NISTIR 5477

Sharma, U. et al. 2002, these proceedings

Skinner, C. J., Bergeron, L. E., \& Daou, D. 1997, "Characteristics of the NICMOS Detectors", in HST Calibration Workshop, STScI, Baltimore, MD, 171

Terrestrial Planet Finder, Origins of Stars, Planets, and Life, eds. C. A. Beichman, N.J. Woolf, C.A. Lindersmith,, JPL Pub. 99-3, 1999, see http://tpf.jpl.nasa.gov/

Wilson, J. W., Townsend, L. W., Healy, J.E., Chun, S.Y., Hong, B.S., Buck, W.W., Lamkin, S.L., Banapol, B.D., Khan, F., \& Cucinotta, F.A. 1989, “BRYNTRN: A Baryon Transport Model,” NASA Technical Paper 2887 